\title{
La puntura ad occhiello della FAV arterovenosa. Storia e dati di evidenza nell'applicazione della tecnica
}

\section{Pegoraro}

Infermiera, CAL Corsico; U.O. Ospedale Niguarda Ca' Granda, M ilano

EDTNA-ERCA Education Board, EDTNA-ERCA Filiale Italiana

\section{Introduzione}

$\mathrm{Nel} 2004$, in una sessione congressuale dell'EDTNAERCA, un collega belga, presentò in dettaglio l'applicazione della tecnica di "puntura ad occhiello" per la venipuntura delle FAV native in pazienti in emodialisi domiciliare e in assistenza limitata. N ella stessa sessione, una seconda relazione, di una collega canadese, riportava i dati dell'applicazione della stessa metodica su FAV in condizioni cliniche precarie. In entrambi i casi, i dati erano molto interessanti.

Dopo essere stata per anni quella "dei buchi nuovi" amata e odiata dai pazienti, sentivo di avere trovato un'equa risposta alla considerazione clinica di contenere la formazione delle dilatazioni aneurismatiche delle FAV, provocando il minore dolore possibile all'atto della venipuntura.

Cercai quindi l'occasione di recarmi a Bruxelles a visitare il Centro del collega per raccogliere e vagliare i dati esperienziali e personali sull'applicazione della tecnica, che nel frattempo era stata estesa anche ai pazienti in emodialisi ospedaliera, di maggiore età anagrafica e dialitica. M ediamente le braccia di quei pazienti erano in buone condizioni, sicuramente meno bitorzolute di quelle dei pazienti di pari età, che ero solita vedere. Lavorando da molto tempo in un CAL sono sempre stata attenta all'accesso vascolare dei miei pazienti: I'esperienza belga ha attivato il mio interesse per questa tecnica e per la sua conoscenza anche nell'ambito dialitico nazionale.

\section{Le origini}

Il padre indiscusso della puntura ad occhiello è il dottor T wardowsky che sin dai primi anni Settanta quando ancora lavorava in Polonia, applicò su alcuni pazienti, che avevano limitati punti di repere della FAV, quella che lui chiamava la tecnica di "puntura a sito costante", in alternativa alla rotazione costante dei siti. Aveva potuto notare come, mantenendo costante il sito di inserzione degli aghi, non comparissero gli aneurismi, il dolore riferito fosse molto inferiore el'incidenza di problematicità fosse anche minore rispetto alle altre tecniche (1). Tali dati vennero poi confermati da Kronung, nei primi anni Ottanta (2), il quale condusse degli studi sull'emodinamica e sulle leggi fisiche che intervengono, e sono conseguenti, alla creazione di una FAV, al suo sviluppo e alla deformazione aneurismatica dei vasi interessati. A lui si deve la famosa figura di come si sviluppano i vasi in relazione alle metodiche di puntura usate, così come la nomenclatura e le definizioni dei vari risultati (Fig. 1): puntura a "scala di corda", per definire la rotazione dei siti di inserzione degli aghi; puntura ad "area", per definire le punture concentrate in zone circoscritte e limitate; puntura ad "occhiello", per definire la tecnica di puntura a sito costante. I dati pubblicati da Kronung affermano che: a) i vasi arterializzati sono sottoposti nel tempo a una fisiologica dilatazione data dall'aumento della portata e della pressione; b) a ogni puntura consegue la creazione di un tessuto cicatriziale che rispetto al tessuto sano è meno elastico, più fibroso, meno capace di contenere la pressione presente nel vaso; c) i vasi si sviluppano e si modificano in base a come sono ripetutamente punti; d) la puntura ad "area" è quella che maggiormente induce aneurismi e stenosi concomitanti, secondo la legge dell'emodinamica di Bernulli, li amplifica esponenzialmente in base a velocità e pressione del flusso (Fig. 4); e) la tecnica che induce una uniforme dilatazione lungo tutto il decorso del vaso, senza creare aneurismi, è quella "a scala di corda" (Fig. 2); f) la tecnica migliore era quella a sito costante, "occhiello" perché non induceva la dila- 


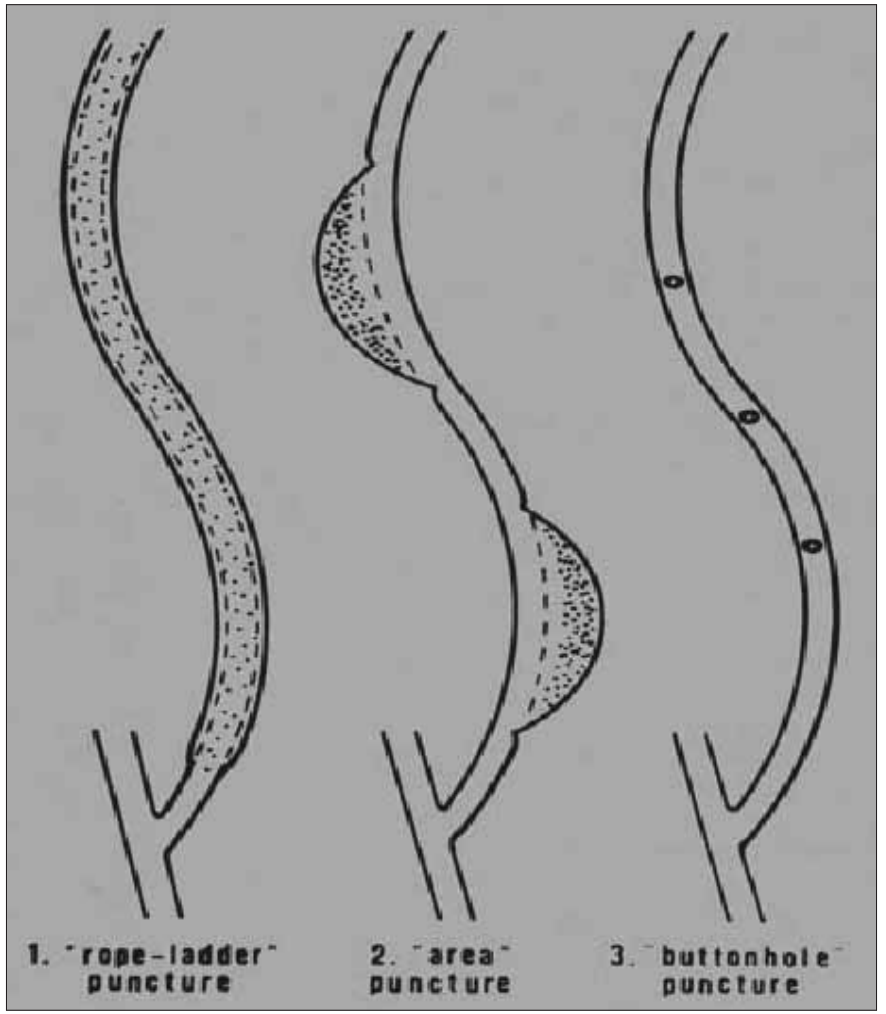

Fig. 1 - 1) Puntura a scala di corda; 2) Puntura ad area; 3) Puntura ad occhiello.

tazione dei vasi (Fig. 3). Nella stessa pubblicazione ci sono dati relativi alla puntura delle FAV protesiche, che non sono oggetto della presente trattazione.

$M$ algrado i dati e nonostante tutte le positive ricerche empiriche, la puntura ad occhiello non ha avuto alcun seguito in Italia, e nei Paesi del Sud Europa: è invece diventata progressivamente una tecnica usata nei Centri dialisi nei Paesi del Nord Europa e del Nord America (dopo che T wardowsky si spostò in Canada).

La letteratura scientifica sui criteri di puntura delle FAV risulta, in genere, scarsa e i contributi degli autori citati mantengono nel tempo originalità ed efficacia. Le ragioni per tale vuoto sono ricollegabili a una concomitanza di situazioni: quella etica che ha concentrato gli sforzi sulle priorità di provvedere al trattamento dialitico per tutta la popolazione; quella tecnica del curare l'efficienza attraverso la tecnologia (membrane, filtri, metodiche); quella socio-culturale intesa come una scarsa attenzione verso le priorità espresse dai singoli pazienti (la conservazione dell'immagine corporea, il contenimento del dolore). In ultima analisi anche il fatto che la metodica appar tenesse alle competenze infermieristiche e che le infermiere scri-

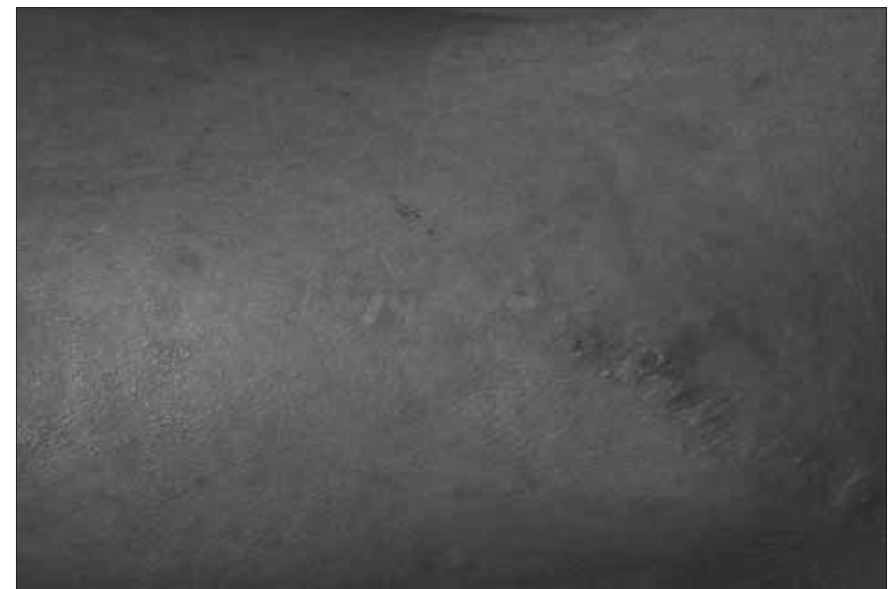

Fig. 2 - Esempio di puntura a scala di corda.

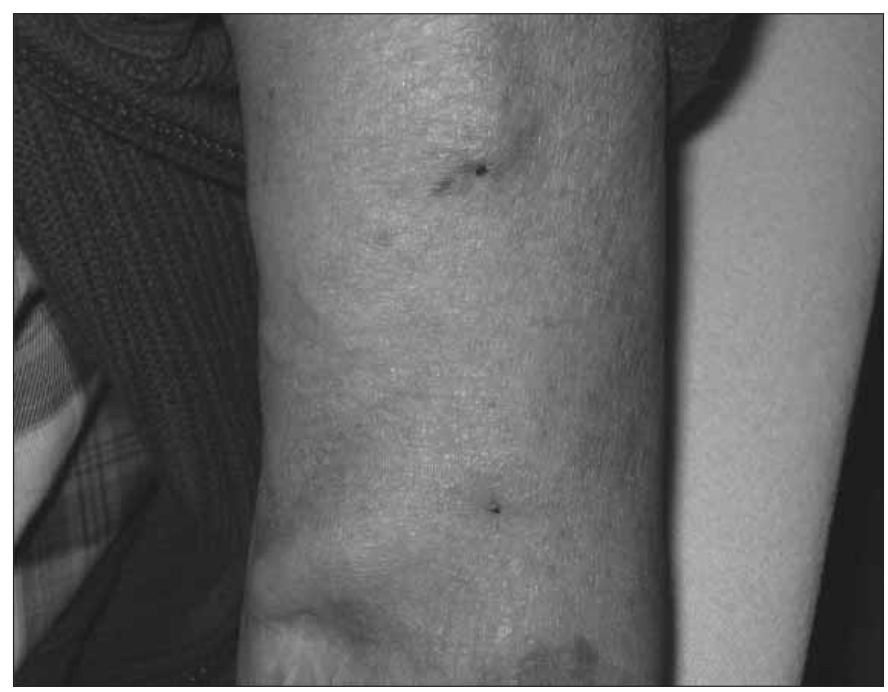

Fig. 3 - Esempio di puntura ad occhiello.

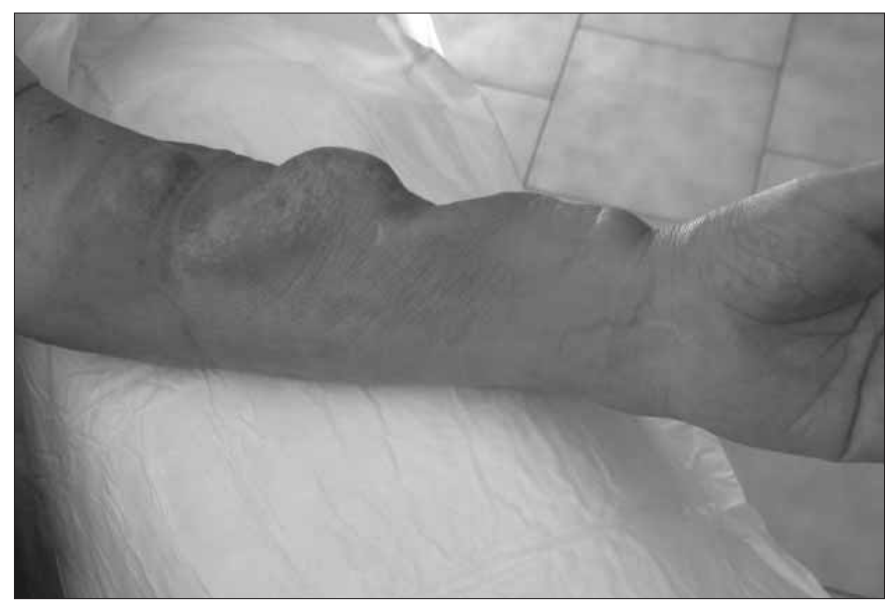

Fig. 4 - Esempio di puntura ad area. 
vessero poco in genere e, ancor meno, sul loro modo di operare nella pratica clinica.

In questi ultimi anni l'atteggiamento sociologico, etico e di qualità delle cure si è progressivamente spostato verso l'attenzione alla persona. Nel contempo le infermiere hanno, in genere, elevato il loro livello culturale e la loro autonomia decisionale, sentendosi in grado ed avendo l'esigenza di esprimere, confrontare dati circa la tipologia e la qualità del loro esercitare. Non ultimo, a seguito dei dati DOPPS di questa ultima decade, è risultato evidente che: la FAV nativa è l'accesso vascolare di eccellenza per il paziente in trattamento emodialitico, indipendentemente da età e co-morbidità e che i dati europei di pratica e gestione degli accesi vascolari avevano risultati di durata ed efficienza notevolmente migliori di quelli americani.

I dati empirici e di evidenza hanno fatto sì che già le Linee Guida KD QI-2005 (6) indicassero la puntura ad occhiello (Button/ Hole) come quella che meglio preser vava la vita delle FAV native nel tempo. Per quelle protesiche la tecnica di puntura consigliata rimane quella "a scala di corda", I'applicazione della tecnica ad occhiello è ancora sperimentale e non sono ancora stati pubblicati dati di evidenza.

\section{II metodo}

I cardini della tecnica di puntura ad occhiello (Tab. I) delle FAV native sono:

1. Ia personalizzazione dei siti di puntura: per ogni paziente vengono individuati almeno due siti di inserzione, uno per l'ago; "arteria", di aspirazione, e uno per l'ago "vena", rientro;

2. alla dialisi successiva, prima di re-inserire l'ago è d'obbligo l'asportazione della crosta creatasi dalla precedente puntura e il reinserimento dell'ago esattamente nello stesso sito precedente;

3. Ia possibilità di utilizzare un ago smusso, ma non tagliente, quando la tecnica è assestata.

Per i singoli punti citati è necessario inoltre chiarire che:

1) con il termine di personalizzazione si intende che per ogni paziente vengono individuati dei siti di puntura e che gli aghi saranno inseriti esattamente in quei siti, a ogni dialisi. I criteri della scelta del sito sono:

- cute integra (il più possibile), non aree aneurismatiche. N elle FAV già usate è importante scegliere le aree in cui la cute non sia francamente cicatriziale e abbia perso l'elasticità naturale;

- assenza di situazioni infiammatorie, dermatologiche, trombotiche;

- distanza di almeno 5-7 centimetri tra i siti di venipuntura, se posti sulla stesso vaso.
Nella fase iniziale di puntura dei siti appena definiti è importante che un solo operatore (il paziente, il partner o un infermiere) operi la procedura di venipuntura, in modo da permettere un gesto il più ripetitivo possibile. $\mathrm{N}$ ei casi in cui questo non sia possibile è importante definire un numero limitato di infermieri (non più di tre) atti alla venipuntura almeno nelle prime 2-3 settimane. D opo questo periodo la microstruttura cicatriziale sottocutanea che si sarà formata permetterà l'uso dell'ago smusso in quel punto, e solo in quel punto. Potranno essere istruiti anche gli altri infermieri alla puntura sul sito.

2) Prima di ogni venipuntura bisogna assolutamente togliere la crosta formatisi dalla puntura precedente. Questa manovra è lontana dalla usuale prassi e incontra spesso molte resistenze psicologiche o pratiche da parte degli operatori. Questa manovra serve essenzialmente per:

- portare allo scoper to la microarea di sottocute cicatriziale che è più fragile, meno inquinata;

- esaltare il punto di inserzione e averlo il più "definito" possibile: è imperativo andare esattamente sullo stesso sito. È importante vedere bene e nitido per essere precisi. Questa precisione non è richiesta con le altre tecniche e può essere inficiata da una presbiopia fisiologica, non ancora rilevata, dell'operatore o del paziente che si autopunge;

- ridurre al minimo il rischio di introdurre all'interno del vaso il materiale esterno, normalmente contaminato dalla flora cutanea residente;

- occludere parzialmente il calibro interno dell'ago con del materiale biologico.

La crosta viene ammorbidita lasciando in sito le garze intrise di soluzione fisiologica o disinfettante normalmente usato per le FAV, prima dell'inizio della seduta. La crosta viene rimossa facilmente e in modo sicuro con un ago sterile monouso 19/20 G, usandolo parallelamente alla cute, facendo leva su un bordo della crosta con una micro manovra di leva.

È fondamentale NON USARE gli stessi aghi da FAV per rimovere la crosta. La ragione è chiara, ma la "tentazione" per gli operatori meno motivati è forte, ma decisamente deleteria per l'elevato rischio di inquinamento dell'ago e rischio di infezione dell'area. Alla rimozione della crosta segue la ri-disinfezione del sito.

L'inserzione dell'ago nello stesso sito induce a ogni successiva puntura una meno intensa sensazione del dolore percepito dal paziente, riferito dai più come il sentire "una pressione" più che un dolore. Ciò è da attribuirsi alla recisione dei microrecettori cutanei, a seguito del ripetersi delle punture. 
TABELLA I - PROCEDURA DI ATTUAZIONE DELLA PUNTURA AD OCCHIELLO

\section{1) Selezione dei siti più idonei}

- cute elastica;

- tratto retto del vaso;

- idoneità di eventuale autopuntura;

- aree non infiammate;

- non cicatriziali, o già troppo usate.

\section{2) Disinfezione della cute}

Usare il disinfettante e la tecnica normalmente utilizzati: nel vostro Centro.

\section{3) Rimozione delle croste}

- insegnare al paziente a lavarsi il braccio a casa e cercare di ammorbidire o eliminare (per quanto possibile) le croste;

- posizionare garze intrise di disinfettante, per un tempo un po' maggiore del solito, prima dell'inizio della seduta, sulle aree di puntura, per ammorbidire le croste;

- usare un ago sterile da intramuscolo/ endovena (19/20G) per eliminare i residui di croste;

- assolutamente non usare l'ago da fistola per la loro rimozione;

- verificare se nella vostra prassi vi sono situazioni specifiche che possono beneficiare di altre strategie (ad esempio, cerottino con pomata anticoagulante, da mettere sulla crosta prima di uscire di casa per andare in dialisi).

4) Ri-disinfezione dei siti di puntura

Importante, da non tralasciare.

Usando la garza già presente nel vostro piano di lavoro.

\section{5) Inserimento degli aghi esattamente nello stesso punto in cui vi erano le croste}

È importante andare esattamente nello stesso punto.

Se vi accorgete di vedere sfuocato, non chiaro, mettete gli occhiali per questa manovra.

\section{6) Usare per 6-8 sedute di dialisi gli aghi normali}

In questo periodo, possibilmente un solo pungitore, 0 un numero limitato di pungitori, tra loro in sintonia.

II periodo può variare rispetto ai pazienti e alla tipologia del sito.

7) Passare all'uso dell'ago non tagliente (Bio-hole, Nipro Italia)

M odesta variabilità nella durata di questa fase, dovuta a variabili del paziente quali: tipologia del sottocute, età anagrafica, comorbidità. Se risultasse ancora difficile, ridisinfettare e mettere una ago normale, esattamente nello stesso punto.

Su una modesta percentuale di pazienti non è sempre possibile usare l'ago smusso, per la presenza di un "effetto rimbalzo" o di un sottocute fibroso. II sito di puntura può essere mantenuto costante continuando a usare i nor mali aghi da FAV.

La manualità dell'uso degli aghi smussi si acquisisce con un po' di pratica e perseveranza.

8) Estendere l'incannulazione del vaso a tutti gli operatori

- previa informazione e tutoraggio;

- aderenza alle procedure codificate.

3) Dopo aver punto per 6/ 8 sedute con un ago normale da emodialisi (tagliente da 16 o $15 \mathrm{G}$ come da prassi del Centro), nei siti di puntura si formerà un invisibile tunnel cicatriziale, definibile anche come "un invito" di inserimento, che indica il sito di inserzione e permette, in quella specifica microarea, l'uso dell'ago smusso. L'ago smusso non recide, bensì sposta le fibre cicatriziali più lasse senza tagliarle e arrivato in prossimità della parete venosa si rende necessaria una manovra di leggera rotazione e pressione, per permettere all'ago di posizionarsi nel punto di minore resistenza ed entrare nel vaso. L'utilizzo dell'ago smusso neces- sita di una manualità percettiva leggermente diversa dagli aghi taglienti, specificità che viene acquisita con l'uso del presidio. A fine trattamento l'ago viene rimosso con la solita accortezza: seguire il medesimo angolo di entrata, premere solo quando l'ago è fuoriuscito. II tessuto sottocutaneo circostante essendo sano, tonico, ancora elastico, si richiude intorno al sito di inserzione. L'emostasi è mediamente più breve di quanto accada con le altre tecniche di puntura. La crosta che consegue l'uso degli aghi smussi è più piccola e leggermente introflessa. In una percentuale di casi (dal 5 al 15\%), nelle FAV di grande portata e già usate 


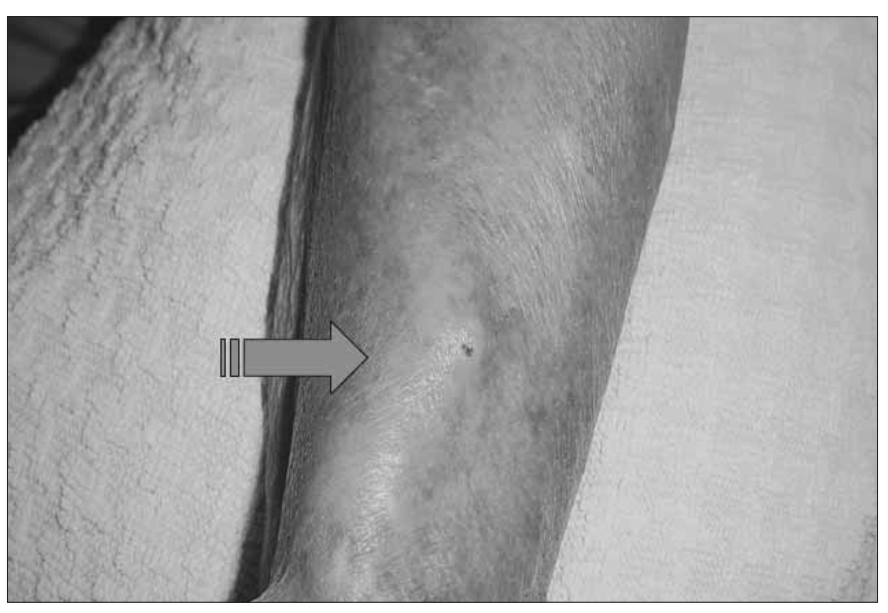

Fig. 5 - In paziente non giovane, con sottocute molto sottile, 53 mesi di puntura ad occhiello su FAV prossimale.

da tempo, o rivisitate da inter venti riparativi, la parete vasale crea un "effetto rimbalzo" che spinge l'ago verso l'esterno. In questi casi si può mantenere costante il sito di inserzione con la puntura ad occhiello, continuando a usare i normali aghi da FAV. La tecnica di puntura ad occhiello può essere attuata anche continuando a usare gli aghi taglienti, fermo restando che, poiché l'ago taglia, è in grado di ledere il micro tessuto cicatriziale nel punto di inserzione, e quindi sarà potenzialmente più facile avere delle conseguenti micro perdite ematiche intorno all'ago durante la seduta. La corretta applicazione della procedura e l'attenzione dell'operatore assicurano il persistere di buoni risultati e l'efficacia della tecnica.

\section{Discussione}

Una ricerca multicentrica che l'EDTNA-ERCA (associazione europea di infermieri di dialisi e trapianto) ha attivato sulla sorveglianza e gestione degli AV nei Centri dialisi europei, svoltasi tra il 2000 e il 2002 con il coinvolgimento di 108 Centri in 18 diversi Paesi, ha evidenziato come la gestione degli accessi vascolari, in genere, sia un'attività di competenza infermieristica. In sempre maggiori realtà internazionali, questo dato viene confermato dalla creazione di ruoli infermieristici clinici dedicati alla gestione degli accessi vascolari nei singoli Centri (vascular access nurse), con attività di coordinamento, formazione continua del personale, cooperazione con gli specialisti vascolari e inter ventistici, al fine di dare continuità clinica, gestionale e qualitativa all'attività relativa agli accessi vascolari.

La tecnica di puntura a sito costante, o tecnica ad oc-

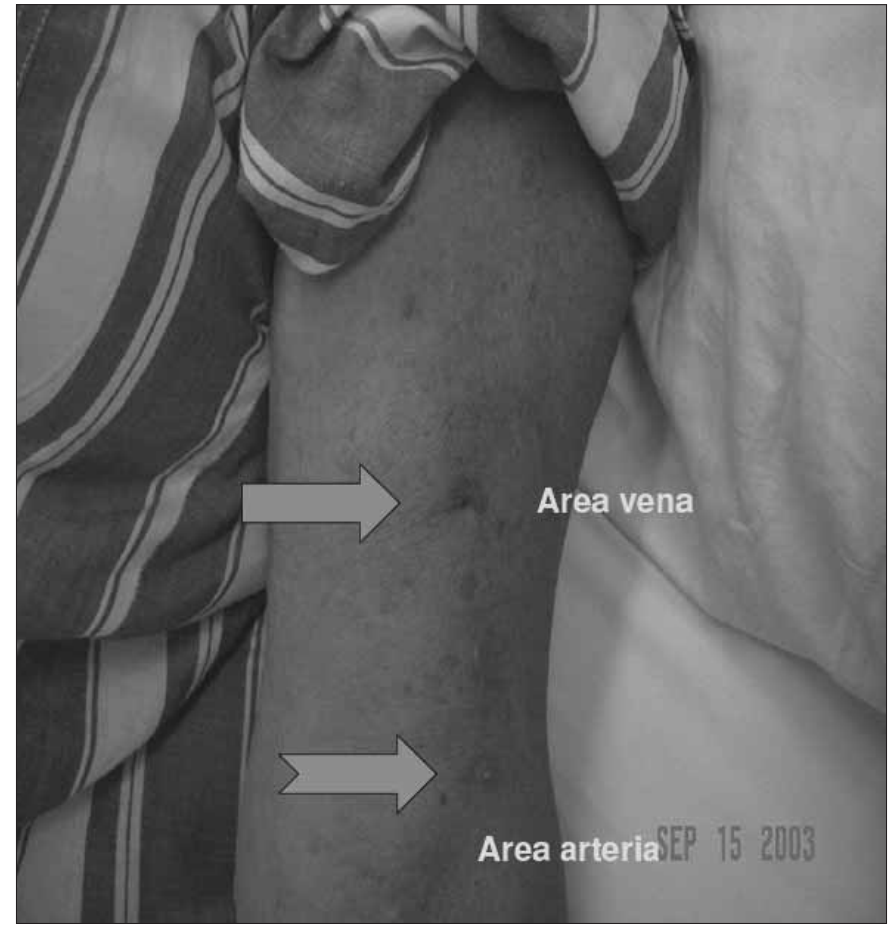

Fig. 6 - Esempio di FAV prossimale punta ad occhiello.

chiello, ha dato ottimi risultati di performance nei Centri che I'hanno adottata. Può essere applicata sia nelle FAV di nuova confezione (4), sia in quelle già usate (5) e critiche, fermo restando alcuni criteri pratici da rispettare nell'uno e nell'altro caso. Benché la tecnica di puntura a scala di corda sia stata considerata dal pensiero comune come la migliore, nella realtà operativa, viene disconosciuta perché agli infermieri non piace sbagliare (4): la rotazione dei siti implica venipunture in aree a rischio e non tutti gli operatori hanno le necessarie abilità; i pazienti non vogliono sentire male né gradiscono i "buchi mancati". Ne consegue che la tecnica più applicata è quella ad "area", con una notevole diffusione delle classiche due, o più, aree dilatate e le inevitabili stenosi che, a seconda delle tipologie del sottocute del paziente, portano alla morbilità/ mortalità dell'accesso. Se a questo aggiungiamo un soggettivo sconfor to della persona dato dal deturpamento estetico dell'arto e dal dolore fisico alle singole inserzioni degli aghi, ne consegue una valutazione qualitativa tecnico-relazionale bassa o con ampio margine di miglioramento.

L'implementazione della tecnica nei Centri dialisi, in genere, incontra una certa resistenza sostenuta più dalla difficoltà di abbandonare le consuetudini che da vere motivazioni scientifiche. Dopo una accurata valutazione dei dati di evidenza raccolti e presentati dai Centri che attuano da tempo questa tecnica per la puntura delle FAV 
native, i motivi per non provare appaiono spesso inconsistenti (Figg. 5, 6).

L'applicazione di questa tecnica è più facile nei Centri dove esista già una buona attenzione alla gestione delI'accesso e una modulistica specifica di mappatura e monitoraggio. Allo stesso modo l'implementazione di un approccio strutturato alla venipuntura della FAV può essere un valido stimolo alla creazione di strumenti di gestione e monitoraggio qualora non siano stati già sviluppati.

$\mathrm{Nel}$ singolo Centro, la tecnica può essere iniziata con pochi pazienti, magari quelli più complianti, oppure con le FAV che di base non hanno problemi funzionali tali da poter poi dare dei falsi negativi nella valutazione dell'efficienza della tecnica. O ppure potrebbe essere iniziata su FAV che davvero hanno poco spazio di accesso al vaso e dove la criticità all'incannulazione e/ o alla formazione di aneurismi siano di fatto elevate.

\section{Conclusioni}

La criticità maggiore per l'applicazione della tecnica non è il paziente, ma piuttosto il personale infermieristico e medico. Nel primo per la necessità di un maggiore coinvolgimento e attenzione nella fase di puntura, soprattutto nel periodo iniziale, ma anche nel prosieguo, da parte di tutto il gruppo di lavoro, che necessita di una informazione precisa sull'esecuzione della procedura, e la formazione corretta del personale nuovo (3). Alcune problematicità emerse negli incontri e nei "luoghi comuni" del pensiero, non sono imputabili alla tecnica in se stessa, ma alla scorretta o non adeguata applicazione delle procedure da par te degli operatori. La criticità nella fase iniziale, dovuta alla limitazione del numero delle persone che devono pungere, può essere superata con una riorganizzazione delle competenze e delle presenze alI'interno del gruppo infermieristico; la necessaria aderenza alla procedura quando la tecnica sia stabilizzata (disinfezione/ rimozione della crosta/ disinfezione) può essere sostenuta con l'implementazione di una adeguata documentazione dedicata all'accesso vascolare. Si ribadisce la facilità del mantenimento della tecnica e la possibilità per i pazienti idonei di autopungersi, nell'intento di riabilitare e rendere i soggetti, quando possibile, più consapevoli del loro corpo e magari anche del loro trattamento/ salute.

Da parte del personale medico le reazioni percepite dalla scrivente con maggiore frequenza sono una certa diffidenza nel considerare il valore di dati provenienti da un altro gruppo professionale, un eccesso di zelo nel valutare e considerare criticamente, un approccio nuovo e strutturato, in contrasto con un cer to disinteresse per la prassi della venipuntura di qualità dell'accesso periferico in genere.

Con ragionevole certezza dettata da anni di esperienza consolidata in molti grandi e piccoli Centri, la tecnica di puntura ad occhiello fornisce un sicuro e confermato beneficio per il paziente nel breve, medio e lungo periodo. Sta a noi operatori trovare le strategie per implementare al meglio nella nostra realtà, i miglioramenti che l'esperienza propone.

\section{Note}

L'autore si rende disponibile a chi desideri ulteriori approfondimenti sia per singolo Centro sia per gruppo di Centri limitrofi, con l'obiettivo di diffondere e sostenere l'applicazione della tecnica di puntura ad occhiello e con I'intento di raccogliere dati sull'utilizzo di tale tecnica. Coloro che fossero interessati a una o a entrambe le iniziative possono contattare direttamente l'autore presso: Cal Corsico tel. 026444 5091, fax 5092,

e-mail: marisa_pegoraro@fastwebnet.it

\section{B ibliografia}

1. Twardowsky Z, Kubara H. Different sites versus constant site of needles insertion into arteriovenous fistulas for treatment by repeated dialysis. Dial Transplant 1979; 8: 978-80.

2. Kronung G. Plastic deformation of Cimino fistola by repeated puncture. Dial Transplant 1984; 13: 635-8.

3. Ball LK. The button hole technique for arterovenous fistulas cannulation. NNJ 2006; 33: 299-304.

4. Goovaerts T. Presentazione alla conf. Inter. EDTNAERCA, Ginevra; 2004.

5. M articorena R. Presentazione alla conf. Inter. EDTNAERCA, Ginevra; 2004.

6. NKF K/DOQI GUIDELINE 3. Cannulation of fistula and graft's and accession of hemodialysis cathethers and port cathether systems, updates 2006. 